\title{
Ivabradina, insufficienza cardiaca e malattia renale cronica
}

\author{
Giovanni Barbera ${ }^{1}$, Alberto Santoboni ${ }^{1}$, Antonio De Pascalis ${ }^{2}$, Rodolfo Rivera ${ }^{3}$, Fulvio Floccari ${ }^{4}$, Claudio Ronco ${ }^{5}$, Luca Di Lullo ${ }^{1}$ \\ ${ }^{1}$ U.O.C. Nefrologia e Dialisi, Ospedale L. Parodi-Delfino, Colleferro (RM) \\ ${ }^{2}$ U.O.C. Nefrologia, Dialisi e Trapianto, Ospedale V. Fazzi, Lecce \\ ${ }^{3}$ U.O.C. Clinica Nefrologica, Ospedale S. Gerardo, Monza (MB) \\ ${ }^{4}$ U.O.C. Nefrologia e Dialisi, Ospedale S. Paolo, Civitavecchia (RM) \\ ${ }^{5}$ International Renal Research Institute, Ospedale S. Bortolo, Vicenza
}

\begin{abstract}
Ivabradine, heart failure and chronic kidney disease
Incidence and prevalence of congestive heart failure are raising, especially in western countries. In Europe and in the United States it represents a disabling clinical disease for all injured people and it's accountable for the increase of hospitalization and sanitary costs.

European guidelines have underlined the importance of the optimization of pharmacological treatment to improve both patients' outcomes and quality of life.

SHIFT trial's results, based on the evaluation of ivabradine's efficacy if added to conventional congestive heart failure's therapy, seem to proceed in that direction.
\end{abstract}

Keywords: Heart failure, Comorbidities, Ivabradine, SHIFT trial, Morbidity, Mortality, Hospitalizations, Ventricular remodelling, Beta-blockers

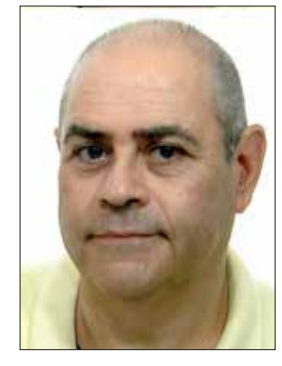

Giovanni Barbera

\section{Introduzione}

Lo scompenso cardiaco (SC) rappresenta attualmente una delle principali cause di decesso per malattia cardiovascolare tra le popolazioni del mondo occidentale. La mortalità negli individui di oltre 65 anni si aggira intorno al 50\%, nonostante i progressi ottenuti a partire dagli anni '90 a seguito dell'introduzione in terapia dei farmaci attivi sul RAAS e dell'uso dei beta-bloccanti.

L'incidenza attuale dello scompenso cardiaco tende ad aumentare, in rapporto con le fasce d'età, partendo da 2.5/1000 persone/anno nella fascia d'età compresa tra i 55 e i 64 anni sino ad arrivare a 44/1000 persone/anno per la fascia d'età dagli 85 anni in su.

Ai nostri giorni, la prevalenza di insufficienza cardiaca sin-

Accepted: May 21, 2015

Published online: June 5, 2015

Indirizzo per la corrispondenza:

Dr. Luca Di Lullo

U.O.C. Nefrologia e Dialisi

Ospedale L. Parodi-Delfino

Piazza A. Moro 1

00034 Colleferro (RM)

Dilulloluca69@gmail.com tomatica nella popolazione del nostro continente oscilla tra lo $0.4 \%$ e il $2 \%$ per le fasce d'età intermedie ( $5^{a}-6^{a}$ decade), potendo arrivare a superare il $10 \%$ nei soggetti oltre i 70 anni. Una quota variabile intorno all' $1.5 \%$ dei soggetti è, peraltro, affetta da forme asintomatiche di scompenso cardiaco (1).

Questo fa sì che lo scompenso cardiaco sia tra i principali motivi di ospedalizzazione oltre i 65 anni di età, rappresentando un impegno sia in termini di risultati clinici, per gli outcome altamente invalidanti, che in termini di economia sanitaria, per l'incremento della spesa pro-capite in tale tipologia di pazienti.

Lo SC rappresenta l'1-2\% di tutta la spesa sanitaria dei paesi occidentali; nel nostro paese, la spesa totale (assistenza ospedaliera, ambulatoriale e domiciliare) per SC è pari all'1.8$2.2 \%$ circa della spesa sanitaria globale (dati del Ministero della Salute) e all' $1.4 \%$ della spesa sanitaria nazionale.

Le patologie cardiovascolari che conducono allo sviluppo dell'insufficienza cardiaca sono numerose, tuttavia, tra di esse, la causa principale di SC è rappresentata, in 2 casi su 3, dalla cardiopatia ischemica.

La terapia si è giovata, nel corso degli ultimi 30 anni, dell'introduzione di sempre nuove molecole atte alla cura delle acuzie (p. es., il supporto inotropo nella grave insufficienza cardiaca congestizia acutamente scompensata con Levosimendan) ma anche per il supporto della cronicità della malattia.

Recenti studi hanno posto in evidenza l'importanza dell'aumento della frequenza cardiaca (FC) quale fattore prognostico 
negativo nel decorso della patologia cardiovascolare, compreso lo SC (2-5).

Tali osservazioni costituiscono il razionale del ricorso ai farmaci beta-bloccanti i quali, riducendo, come noto, la frequenza cardiaca, riducono il consumo di ossigeno e il dispendio energetico del miocardio, soprattutto e principalmente nelle forme a genesi ischemica.

L'introduzione della terapia beta-bloccante nello scompenso cardiaco è uno degli eventi più rilevanti della cultura cardiologica moderna, essendo stato il risultato di una modificazione concettuale radicale nell'interpretazione fisiopatologica della malattia.

Esiste, oggi, una documentata evidenza clinica del fatto che piccole dosi di beta-bloccante, somministrate a posologia cautamente crescente nell'arco di settimane o mesi, possono indurre un significativo miglioramento clinico nella funzione ventricolare sinistra (riduzione della mortalità di circa il $30 \%$ e delle ospedalizzazioni di circa il $40 \%$, miglioramento della classe funzionale del $30 \%$ e incremento della frazione di eiezione di circa il $30 \%$ ) e, secondo alcuni Autori, una riduzione delle dimensioni cardiache, che si mantengono nel tempo, anche se non indefinitamente. Verosimile appare, inoltre, una riduzione della mortalità.

I dati desunti dai grandi trial clinici randomizzati (MDC, CIBIS-II, MERIT-HF) suggeriscono che i pazienti con scompenso cardiaco sistolico, lieve o moderato (classe NYHA I-III), clinicamente stabile, traggono giovamento dalla terapia con betabloccante.

Tuttavia, in una percentuale di pazienti che si aggira intorno al $25 \%$, vi sono chiare controindicazioni al blocco beta adrenergico (p. es., BPCO, arteriopatici, diabetici, ecc.) e non tutti i soggetti sottoposti a terapia con beta-bloccante riescono a raggiungere la dose target ottimale di farmaco a causa della comparsa di effetti collaterali (soprattutto astenia con notevoli limitazioni funzionali, peggioramento degli indici di contrattilità per l'effetto inotropo negativo, ecc.). In particolari categorie di soggetti affetti da SC, pertanto, nonostante una terapia che mira a un dosaggio "on top" di beta-bloccante, non si riesce a ottenere un adeguato controllo della frequenza cardiaca a svantaggio della gittata cardiaca e della forza contrattile di per sé già compromesse, nonché del dispendio energetico del muscolo cardiaco.

La prima evidenza di un'importanza prognostica della frequenza cardiaca sull'outcome delle malattie cardiovascolari risale a uno studio di Levy et al. del 1945 (6), in cui si dimostrava il significato prognostico dell'incremento transitorio della FC da solo e in associazione con l'ipertensione transitoria.

In altri studi, successivamente è stata sottolineata l'associazione tra FC ed eventi cardiovascolari, in particolare con la mortalità (totale, cardiovascolare, improvvisa), in diversi contesti clinici, inclusi l'infarto miocardico (IM) e l'insufficienza cardiaca (IC). La mortalità aumenta, in genere, progressivamente con l'aumento della $\mathrm{FC}$, ma, in alcuni studi, il rischio aumenta maggiormente per $\mathrm{FC}$ superiori a certi valori di cutoff, variabili da 70 a $90 \mathrm{~b} / \mathrm{min}(7-10)$.

In osservazioni recenti, in soggetti affetti da cardiopatia ischemica, è stato evidenziato che un incremento della FC a riposo rappresenta un fattore predittivo indipendente di mortalità cardiovascolare e di aumento del rischio di comparsa di eventi cardiovascolari maggiori nei pazienti con coronaropatia in fase stabile $(11,12)$.

Normalmente, il miocardio ha un dispendio energetico pari a circa $30 \mathrm{~kg}$ di ATP al giorno, per cui una riduzione della FC di $10 \mathrm{bpm}$ permetterebbe il risparmio di ben $5 \mathrm{~kg}$ di ATP al giorno (13).

Dal momento che normalmente il flusso coronarico si verifica in fase diastolica, ciò che pilota il sangue verso le regioni subendocardiche è la differenza tra la pressione arteriosa vigente nelle coronarie e la pressione telediastolica (LVEDP) nel ventricolo sinistro. Pertanto, se il tempo di diastole si riduce dell' $1 \%$, il flusso nelle regioni subendocardiche può incrementarsi dal $2.6 \%$ al $6 \%$.

\section{Ivabradina}

L'ivabradina è un farmaco che riduce in modo selettivo la frequenza cardiaca, agendo attraverso un'inibizione selettiva e specifica della corrente pacemaker cardiaca I $f$, che controlla la depolarizzazione diastolica spontanea nel nodo del seno e che regola la frequenza cardiaca. Gli effetti cardiaci sono specifici per il nodo del seno senza effetti sui tempi di conduzione intra-atriale, atrioventricolare o intraventricolare né sulla contrattilità miocardica o sulla ripolarizzazione ventricolare.

L'ivabradina agisce sulla corrente ionica If $(f$ sta per "fun$n y^{\prime \prime}$, così definita perché ha proprietà non usuali se confrontata con gli altri sistemi ionici noti al tempo della sua scoperta), la quale è altamente espressa nel nodo senoatriale. If è una corrente ionica $\mathrm{Na}^{+}-\mathrm{K}^{+}$in ingresso nelle cellule pacemaker, la quale viene attivata dall'iperpolarizzazione e modulata da parte del sistema nervoso autonomo. Essa rappresenta una delle correnti ioniche più importanti per la regolazione dell'attività di pacing del nodo senoatriale. L'ivabradina, inibendo selettivamente la corrente If del pacemaker in maniera dosedipendente, provoca il blocco di questo canale, riducendo l'attività del pacemaker cardiaco, e, rallentando la frequenza cardiaca, permette al cuore di avere più tempo a disposizione per il flusso intramiocardico $(14,15)$.

L'esclusività del legame tra ivabradina e canale If ha come risultato una riduzione "pura" della FC senza incidere sulla contrattilità miocardica.

Il risultato finale è una riduzione della pendenza del tempo di depolarizzazione diastolico a cui segue la riduzione della FC che si traduce in un prolungamento medio del tempo di diastole di circa 6 minuti all'ora (16-18).

L'ivabradina ha avuto indicazione per l'impiego in soggetti adulti con CAD in normale ritmo sinusale e FC basale oltre i 70 bpm che non siano tolleranti o che abbiano controindicazioni all'uso dei beta-bloccanti o in associazione con questi, in pa- 
zienti inadeguatamente controllati con una dose ottimizzata di beta-bloccante e in soggetti affetti da scompenso cardiaco in classe NYHA dalla $2^{\text {a }}$ alla $4^{a}$ con disfunzione sistolica, in ritmo sinusale e con FC basale superiore a $75 \mathrm{bpm}$ in associazione con la terapia standard, compresi i beta-bloccanti.

Notoriamente, nei pazienti con insufficienza cardiaca, comunemente si associa una quota di disfunzione renale che, in questi soggetti, rappresenta un forte predittore di mortalità (19).

Il trattamento con ivabradina nello scompenso cardiaco non sembra influire sulla funzione renale e tutti gli outcome cardiovascolari risultano migliorati sia nei soggetti con disfunzione renale che in quelli senza (20).

Essa consentirebbe, in tali contesti patologici, una riduzione della mortalità CV o dell'ospedalizzazione per peggioramento dello scompenso, della mortalità per lo scompenso e della riospedalizzazione per lo scompenso.

Queste osservazioni incoraggerebbero, secondo le revisioni più attuali delle Linee Guida europee ESC, l'introduzione dell'ivabradina sia nei soggetti con cardiopatia ischemica e FC $\geq 70$ bpm sia nei portatori di SC cronico e FC $\geq 75$ bpm, da sola $\mathrm{o}$ in associazione con la terapia tradizionale con betabloccante (21).

\section{Ivabradina e trial clinici}

\section{II trial BEAUTIFUL}

I risultati del trial BEAUTIFUL (Morbidity-Mortality Evaluation of the If Inhibitor Ivabradine in Patients with Coronary Artery Disease and Left-Ventricular Dysfunction) sono stati presentati in occasione dell'ultimo Congresso della Società Europea di Cardiologia (Monaco, 2008) $(22,23)$.

BEAUTIFUL è il primo studio in doppio cieco, randomizzato vs placebo, controllato, che valuta in modo prospettico la correlazione tra frequenza cardiaca ed eventi cardiovascolari, condotto su un totale di 10917 pazienti.

I criteri di inclusione erano fondamentalmente quattro: la presenza di coronaropatia stabilizzata documentata negli ultimi 3 mesi, di disfunzione ventricolare sinistra confermata all'ecocardiogramma, di una frequenza cardiaca a riposo $\geq 60 \mathrm{~b} / \mathrm{min}$ e di ritmo sinusale.

L'endpoint primario dello studio consiste in un endpoint composito di mortalità cardiovascolare, ricovero per infarto del miocardio (IM) e ricovero per insorgenza o peggioramento di insufficienza cardiaca (IC), il quale finisce, quindi, per riunire endpoint cardiovascolari con differente base fisiopatologica. L'obiettivo dello studio è, quindi, la valutazione del beneficio della riduzione della frequenza cardiaca, attraverso l'impiego di ivabradina, su endpoint cardiovascolari di natura diversa.

Sono stati considerati, inoltre, diversi endpoint secondari: mortalità totale, mortalità da cause cardiache, mortalità da cause cardiovascolari o ricovero per IC, ricovero per IM o angina instabile, interventi di rivascolarizzazione coronarica, ricovero per IC e ricovero per IM.

Nel gruppo placebo di BEAUTIFUL, vi è un impiego appropriatamente ampio di farmaci di provata efficacia e va sottolineato che l' $87 \%$ dei pazienti era già in terapia con beta-bloccante, configurandosi, così, uno studio sull'uso concomitante di ivabradina con beta-bloccante in grado di fornire dati ulteriori circa utilità e sicurezza nell'associazione di due farmaci bradicardizzanti.

Milleseicentosettantasei pazienti hanno avuto un evento riconducibile all'endpoint primario; 844 (15.4\%) nel gruppo ivabradina e 832 (15.3\%) nel gruppo placebo [hazard ratio (HR) 1.00, intervallo di confidenza (IC) 95\% 0.91-1.1, $p=0.94$ ). L'effetto dell'ivabradina sull'endpoint primario è stato, quindi, neutro.

L'analisi degli effetti di ivabradina in funzione della causa degli eventi ha evidenziato, però, un beneficio statisticamente significativo per gli eventi "ischemici". Infatti, i ricoveri ospedalieri per infarto miocardico acuto (fatale e non fatale) sono risultati ridotti del $36 \%$ (HR 0.64, IC 95\% 0.49-0.84, $\mathrm{p}=0.001$ ) e le procedure di rivascolarizzazione coronarica del $30 \%$ (HR 0.70, IC 95\% 0.52-0.93, p = 0.016).

\section{II trial SIGNIFY}

II trial SIGNIFY (Study assessInG the morbidity-mortality beNefits of the If inhibitor ivabradine in patients with coronarY artery disease without heart failure) (24) è uno studio in doppio cieco, randomizzato e controllato con placebo con l'ivabradina in aggiunta alla terapia base standard, in 19.102 pazienti con malattia coronarica stabile senza insufficienza cardiaca clinica e con una frequenza cardiaca di 70 battiti al minuto o più (tra cui 12.049 pazienti con angina che limita l'attività fisica [classe $\geq$ Il della scala Canadian Cardiovascular Society, che va da I a IV, con le classi superiori che indicano maggiori limitazioni all'attività fisica a causa dell'angina]).

I pazienti sono stati assegnati a placebo o a ivabradina, alla dose massima di $10 \mathrm{mg}$ due volte al giorno, con la dose regolata per raggiungere una frequenza cardiaca da 55 a 60 battiti al minuto. L'endpoint primario composito era la morte per cause cardiovascolari o l'infarto del miocardio non fatale.

Dopo un follow-up mediano di 27.8 mesi, non vi era alcuna differenza significativa tra il gruppo con ivabradina e il gruppo con placebo nell'incidenza dell'endpoint primario (rispettivamente $6.8 \%$ e $6.4 \%$; hazard ratio, $1.08,95 \%$ intervallo di confidenza, 0.96-1.20, $p=0.20$ ), né vi erano differenze significative nell'incidenza di morte per cause cardiovascolari e infarto miocardico non fatale. L'ivabradina è stata associata a un aumento nell'incidenza dell'endpoint primario tra i pazienti con angina che limita le attività fisiche, ma non tra quelli senza angina che limita le attività ( $p=0.02$ per interazione). L'incidenza della bradicardia è stata più alta con l'ivabradina rispetto al placebo (18.0\% vs $2.3 \%, \mathrm{p}<0.001)$.

Gli Autori spiegano la mancanza di beneficio dell'ivabradina in questo studio con la possibilità che l'ivabradina riduca 
troppo la frequenza cardiaca o che la relazione tra frequenza cardiaca e outcome abbia una curva a forma di J.

In questo trial, tuttavia, sono stati usati i dosaggi più alti del farmaco, rispetto ad altri studi, in associazione con la terapia convenzionale e, inoltre, la bradicardia osservata poteva ascriversi alla concomitante somministrazione di farmaci inibitori del CYP3A4 o di diltiazem o verapamil; escludendo questa serie di pazienti, l'incremento osservato circa il raggiungimento dell'endpoint primario con ivabradina rispetto al placebo appare non significativo (24).

\section{II trial SHIFT}

II trial SHIFT (Systolic Heart failure treatment with the If inhibitorivabradine Trial) (25) è stato eseguito in pazienti affetti da scompenso cardiaco sintomatico con disfunzione sistolica ventricolare sinistra ed è stato condotto in 6.558 pazienti in classe NYHA tra II e IV e con almeno un'ospedalizzazione per riacutizzazione dell'insufficienza cardiaca nell'anno precedente, randomizzati a ricevere ivabradina a una dose iniziale di $5 \mathrm{mg}$ due volte al giorno. Tutti i pazienti erano trattati al top della terapia, con ACE-inibitori o sartani (90\%), beta-bloccanti (90\%) e anti-aldosteronici (60\%), in accordo con le Linee Guida, con un follow-up medio di 23 mesi.

L'endpoint primario composito dello studio consisteva in morte cardiovascolare e ospedalizzazione per riacutizzazione dello scompenso; erano anche considerati degli endpoint secondari, come morte per scompenso cardiaco e ospedalizzazione per scompenso, soprattutto per quanto riguarda $\mathrm{i}$ ricoveri ripetuti per riacutizzazione dello scompenso cardiaco indipendentemente dalla gravità della patologia.

I pazienti che hanno ricevuto ivabradina hanno mostrato una riduzione significativa del $18 \%$ dell'endpoint primario dello studio e una riduzione significativa del $26 \%$ degli endpoint secondari (25).

In una sottoanalisi del trial è stato, inoltre, analizzato l'effetto della terapia con ivabradina in quattro sottogruppi di pazienti: quelli che non assumevano beta-bloccanti, quelli che assumevano dosi di beta-bloccante al di sotto del $25 \%$, tra il $25 \%$ e il $50 \%$ e tra il $50 \%$ e il $100 \%$ e quelli che, invece, raggiungevano la dose target raccomandata dalle Linee Guida.

Nei pazienti che non assumevano i beta-bloccanti o nei due sottogruppi con dose target inferiore al 50\% (dose $<25 \%$ e compresa tra il 25 e il 50\%), si è constatata una riduzione statisticamente significativa dell'endpoint primario composito di morte cardiovascolare e ospedalizzazione per scompenso e di quello secondario, di peggioramento dello scompenso con necessità di ricovero (rispettivamente $p=0.012, p=0.007$ e $p=0.029$ per l'endpoint primario e $p=0.003, p=0.005$ e $p=0.09$ per quello secondario). Nei pazienti che assumevano un dosaggio più elevato di beta-bloccante ( $>50 \%$ della dose target) si è evidenziato un trend di efficacia dell'ivabradina all'aumentare della dose di beta-bloccante (26).

Altri sottostudi (27), in modo particolare quello riguardan- te la qualità della vita e quello riguardante il rimodellamento ventricolare sinistro (SHIFT-ECHO), hanno fatto osservare, nel primo caso (1.944 dei 6.505 pazienti analizzati nel trial principale), una significativa correlazione inversa fra Kansas Score ed eventi clinici, documentando un miglioramento significativo dello score "clinico" (+1.8, $p=0.018)$ e "globale" $(+2.4, p<0.001)$ a 12 mesi. Tali risultati sono in linea con quelli dello studio del gruppo italiano di Gennaro Cice et al. (28-30), CARVIVA-HF, in cui è stato dimostrato che il test del cammino in 6 minuti, la durata al test da sforzo cardio-polmonare, il consumo di ossigeno al picco e la qualità della vita sono migliorati in misura significativa nei pazienti in terapia con la sola ivabradina o con l'associazione ivabradina/carvedilolo, mentre non si sono rilevati cambiamenti di rilievo di questi parametri nel gruppo con il solo carvedilolo.

Nel secondo sottostudio ECHO (411 pazienti dei 6.505 pazienti analizzati nel trial principale), il trattamento con ivabradina ha ridotto, rispetto al placebo, l'endpoint primario di volume telesistolico indicizzato $(-7 \pm 16.3$ vs $-0.9 \pm 17.1$ $\left.\mathrm{mL} / \mathrm{m}^{2}, \mathrm{p}<0.001\right)$ e il volume telediastolico indicizzato $(-7.9 \pm$ 18.9 vs $-1.8 \pm 19 \mathrm{~mL} / \mathrm{m}^{2}, \mathrm{p}=0.002$ ), con un incremento della frazione di eiezione ventricolare sinistra $(+2.4 \pm 7.7 \%$ vs -0.1 $\pm 8 \%$; $<0.001$ ) e una riduzione dei volumi di fine sistole e fine diastole (28-30).

\section{Ivabradina e malattia renale cronica}

Nel 2008, Cice et al. hanno pubblicato dati riguardanti il ruolo della frequenza cardiaca quale fattore prognostico di mortalità in pazienti normotesi sottoposti a dialisi (31).

Lo studio comprendeva 407 soggetti normotesi in dialisi avente come endpoint la mortalità CV e la morte cardiaca improvvisa.

Vennero identificati due cutoff ottimali per la FC >85 bpm e per l'età >65 anni, che si associavano a un incremento del rischio CV, concludendo, pertanto, che l'aumento della FC basale, determinata attraverso un monitoraggio ECG, rappresenta un fattore indipendente di determinismo della mortalità cardiovascolare e globale. II lavoro evidenziava, pertanto, che, nei soggetti in dialisi, vi è una stretta relazione tra sopravvivenza libera da eventi determinanti la mortalità globale e cardiovascolare e livelli basali della FC (31).

II cuore uremico corrisponde, in generale, al paradigma della disfunzione diastolica in cui vari meccanismi come la fibrosi delle fibrocellule muscolari cardiache, lo sviluppo dell'ipertrofia, la silente ischemia, le alterazioni metaboliche e il sovraccarico di calcio conducono ad alterazioni del riempimento diastolico e del rilasciamento ventricolare, nonché alla comparsa di anomalie che compromettono il funzionamento sinergico e sincrono delle pareti ventricolari sinistre.

La disfunzione diastolica del cuore uremico è facilmente rìconoscibile all'eco-color-Doppler cardiaco, attraverso il confronto dei valori di flusso transmitralico e delle velocità al 
TABELLA I - Ivabradina e disfunzione diastolica

\begin{tabular}{lcccc}
\hline & Basale & $\mathbf{1}$ mese & $\mathbf{3}$ mesi & $\mathbf{6}$ mesi \\
\hline IVRT (ms) & $105 \pm 11$ & $96 \pm 14^{*}$ & $76 \pm 12^{*} \S$ & $77 \pm 13^{*} \S$ \\
DT (ms) & $251 \pm 43$ & $230 \pm 37^{*}$ & $207 \pm 43 \S$ & $210 \pm 40^{*} \S$ \\
E/A & $0.6 \pm 0.2$ & $0.8 \pm 0.4^{*}$ & $0.8 \pm 0.4^{*}$ & $0.7 \pm 0.5^{*}$ \\
E/E' & $13.3 \pm 6.4$ & $10.3 \pm 7.1^{*}$ & $9.1 \pm 6.8^{*} \S$ & $9.4 \pm 5.5^{*} \S$ \\
FE (\%) & $56.3 \pm 5.6$ & $57.9 \pm 6.1$ & $57.4 \pm 5.8$ & $58.2 \pm 6.6$ \\
LVMI (g/h2.7) & $56.2 \pm 63$ & $55.4 \pm 6.1$ & $56.5 \pm 5.7$ & $54 \pm 5.7$ \\
\hline
\end{tabular}

IVRT = Tempo di rilasciamento isovolumetrico (v.n.: 60-90), è una misura indiretta del rilasciamento ventricolare. Un suo prolungamento indica un alterato rilasciamento, mentre un suo accorciamento è l'espressione di un aumento delle pressioni di riempimento del VS; DT = tempo di decelerazione dal picco E (v.n.: 182 terza decade, 177 quinta decade, 214 settima decade), rappresenta una misura della funzione diastolica ( ${ }^{*} p<0.05$ vs basale, $\S p<0.05$ vs $\left.1^{\circ} \mathrm{mese}\right)$.

tissue Doppler, permettendo di valutare sia la funzione diastolica che le pressioni di riempimento ventricolare sinistro.

Sempre il gruppo di Cice et al., dell'Università Federico II di Napoli, ha presentato un proprio ulteriore studio su efficacia e tollerabilità del farmaco ivabradina nei pazienti in trattamento dialitico che presentavano un'accresciuta FC basale (32).

Lo studio aveva riguardato un folto gruppo di pazienti in dialisi che manifestavano segni e sintomi di scompenso cardiaco. Su 1241 soggetti selezionati, il 54\% presentava le caratteristiche dello scompenso ipodiastolico, ma vi era anche un discreto numero di soggetti (il 46\%) con le caratteristiche della disfunzione sistolica.

L'88\% dei pazienti studiati si è rivelato in grado di tollerare il dosaggio di $7.5 \mathrm{mg}$ bid.

A 6 mesi, la FC su base media settimanale era stata significativamente minore nel gruppo trattato con ivabradina: valori pre-dialitici (62.4 \pm 7.2 vs $88.3 \pm 6.7 \mathrm{bpm}$; $\mathrm{p}<0.0001)$ e postdialitici (68.1 \pm 8.6 vs $92.5 \pm 13.4$ bpm; $p<0.0001)$ della FC confermano tale andamento.

Tale trend era evidente già dopo 2 settimane e raggiungeva e manteneva il plateau dopo 2 mesi di terapia.

Lo stesso gruppo di lavoro ha valutato, inoltre, gli effetti dell'ivabradina in pazienti in dialisi con scompenso cardiaco congestizio e normale FE. Anche in questo caso, l'effetto dell'ivabradina sulla FC si rivela non difforme da quanto osservato in altre popolazioni di soggetti (p. es., pazienti con scompenso cardiaco ad alta gittata).

In particolare, come mostrato nella Tabella I, nel gruppo di soggetti studiati, il trattamento avrebbe indotto una normalizzazione di alcuni parametri ecocardiografici correlati alla disfunzione diastolica.

Secondo le osservazioni di Cice et al., la terapia con ivabradina avrebbe dimostrato effetti positivi anche sul miglioramento della classe NYHA di scompenso con un progressivo passaggio da classi di gravità maggiore verso classi di gravità minore.

Queste osservazioni vanno a conforto di quanto osservato anche dai ricercatori dello studio SHIFT, secondo i quali il raggiungimento degli endpoint composito primario e secondari era inferiore sia nei soggetti con che in quelli senza disfunzione renale rispetto al gruppo placebo (20).

\section{Conclusioni}

In pazienti affetti da CAD e/o IC in ritmo sinusale la riduzione della frequenza cardiaca si associa alla riduzione della mortalità per cause cardiovascolari. I beta-bloccanti, ampiamente usati nella terapia di tali patologie e indicati dalle recenti Linee Guida, hanno migliorato la prognosi, ma, spesso, vengono usati a dosaggi incongrui (eccessivamente bassi) o scarsamente tollerati dai pazienti.

L'ivabradina da sola o in associazione con i beta-bloccanti è risultata più efficace rispetto ai soli beta-bloccanti nel migliorare la tolleranza all'esercizio fisico e la qualità di vita nei soggetti affetti da CAD e/o IC con FC >70 bpm.

Nei pazienti con scompenso cardiaco, l'ivabradina ha dimostrato di ridurre la mortalità per cause cardiovascolari, la mortalità per scompenso e le ospedalizzazioni per scompenso.

Il suo effetto benefico è presente sia nello scompenso da causa ischemica che in quello da altre cause. Nei pazienti con Insufficienza renale e clearance della creatinina superiore a $15 \mathrm{~mL} / \mathrm{min}$ non sembra necessario alcun adattamento della dose.

Non sono disponibili, in letteratura, dati relativi all'uso dell'ivabradina nei pazienti con clearance della creatinina inferiore a $15 \mathrm{ml} / \mathrm{min}$; anche se recenti osservazioni in soggetti affetti da malattia renale cronica o ESRD sembrerebbero confermarne efficacia e sicurezza, resta consigliato utilizzare una certa prudenza nell'impiego dell'ivabradina in queste tipologie di pazienti.

\section{Disclosures}

Financial support: No financial support was received for this submission.

Conflict of interest: The authors have no conflict of interest.

\section{Bibliografia}

1. Redfield MM, Jacobsen SJ, Burnett JC, et al. Burden of systolic and diastolic ventricular dysfunction in the community: appreciating the scope of the heart failure epidemic. JAMA. 2003;289: 194-202.

2. Jouven X, Empana JP, Schwartz PJ, Desnos M, Courbon D, Ducimetière $P$. Heart-rate profile during exercise as a predictor of sudden death. N Engl J Med. 2005;352:1951-8.

3. Cooney MT, Vartiainen E, Laatikainen T, Juolevi A, Dudina A, Graham IM. Elevated resting heart rate is an independent risk factor for cardiovascular disease in healthy men and women. 
Am Heart J. 2010;159:612-9.

4. Benetos A, Thomas F, Bean K, Albaladejo P, Palatini P, Guize L. Resting heart rate in older people: a predictor of survival to age 85. J Am Geriatr Soc. 2003;51:284-5.

5. Böhm M, Swedberg K, Komajda $M$, et al. The association between heart rate and outcomes in a randomised placebocontrolled trial. Lancet. 2010;376:886-94.

6. Levy RL, White PD. Transient tachycardia. Prognostic significance alone and in association with transient hypertension. Med Press Egypt. 1946;38(6):207-12.

7. Giannoglou GD, Chatzizisis YS, Zamboulis C, Parcharidis GE, Mikhailidis DP, Louridas GE. Elevated heart rate and atherosclerosis: an overview of the pathogenetic mechanisms. Int J Cardiol. 2008;126:302-12.

8. Heidland UE, Strauer BE. Left ventricular muscle mass and elevated heart rate are associated with coronary plaque disruption. Circulation. 2001;104:1477-82.

9. Kannel WB, Kannel C, Paffenbarger RS Jr, Cupples LA. Heart rate and cardiovascular mortality: the Framingham Study. Am Heart J. 1987;113:1489-94.

10. Fox K, Borer JS, Camm AJ, et al. Resting heart rate in cardiovascular disease. J Am Coll Cardiol. 2007;50:823-30.

11. Diaz A, Bourassa MG, Guertin MC, Tardif JC. Long-term prognostic value of resting heart rate in patients with suspected or proven coronary artery disease. Eur Heart J. 2005;26:967-74.

12. Ho JE, Bittner V, Demicco DA, Breazna A, Deedwania PC, Waters DD. Usefulness of heart rate at rest as a predictor of mortality, hospitalization for heart failure, myocardial infarction, and stroke in patients with stable coronary heart disease (Data from the Treating to New Targets [TNT] trial). Am J Cardiol. 2010;105:905-11.

13. Ferrari R. Pathophysiological role of heart rate: from ischaemia to left ventricular dysfunction. Eur Heart J. 2008;10(Suppl. F):F7-10.

14. Tardif JC, Ford I, Tendera M, Bourassa MG, Fox K; INITIATIVE Investigators.Efficacy of ivabradine, a new selective I(f) inhibitor, compared with atenolol in patients with chronic stable angina. Eur Heart J. 2005 Dec;26(23):2529-36.

15. Tardif JC, Ponikowski P, Kahan T. Efficacy of the If current inhibitor ivabradine in patients with chronic stable angina receiving beta-blocker therapy: a 4-month, randomized, placebo-controlled trial. Eur Heart J. 2009;30:540-8.

16. Di Francesco, Camm JA. Heart rate lowering by specific and selective I(f) current inhibition with ivabradine: a new therapeutic perspective in cardiovascular disease. Drugs. 2004;64:1757-65.

17. Thollon C, Cambarrat C, Vian J, Prost JF, Peglion JL, Vilaine JP. Electrophysiological effects of S 16257, a novel sino-atrial node modulator, on rabbit and guinea-pig cardiac preparations: comparison with UL-FS 49. Brith J Pharmacol. 1994;112:37-42.

18. Colin P, Ghaleh B, Monnet X, et al. Contributions of heart rate and contractility to myocardial oxygen balance during exercise. Am J Physiol Heart Circ Physiol. 2003;284:H676-82.

19. Voors AA, van Veldhuisen DJ, Robertson M, et al. The effect of heart rate reduction with ivabradine on renal function in patients with chronic heart failure: an analysis from SHIFT. Eur J
Heart Fail. 2014;16(4):426-34.

20. Tavazzi L, Swedberg K, Komajda M, et al. Clinical profiles and outcomes in patients with chronic heart failure and chronic obstructive pulmonary disease: an efficacy and safety analysis of SHIFT study. Int J Cardiol. 2013;170:182-8. ]

21. ESC Guidelines for the diagnosis and treatment of acute and chronic heart failure 2012: The Task Force for the Diagnosis and Treatment of Acute and Chronic Heart Failure 2012 of the European Society of Cardiology. Developed in collaboration with the Heart Failure Association (HFA) of the ESC. Eur Heart J. 2012;33:1787-847.

22. Tendera $\mathbf{M}$, Talajic $\mathbf{M}$, Robertson $\mathbf{M}$, et al. Ivabradine for patients with stable coronary artery disease and left-ventricular systolic dysfunction (BEAUTIFUL): a randomised, double-blind, placebo-controlled trial. Lancet. 2008;372:807-16.

23. Fox K, Ford I, Steg PG, Tendera M, Robertson M, Ferrari R. Heart rate as a prognostic risk factor in patients with coronary artery disease and left-ventricular systolic dysfunction (BEAUTIFUL): a subgroup analysis of a randomised controlled trial. Lancet. 2008;372:817-21.

24. Fox K, Ford I, Steg PG, Tardif JC, Tendera M, Ferrari R. Ivabradine in stable coronary artery disease without clinical heart failure. N Engl J Med. 2014;371:1091-9.

25. Swedberg K, Komajda M, Böhm M, Borer JS, Ford I, Tavazzi L. Ivabradine and outcomes in chronic heart failure (SHIFT): a randomised placebo-controlled study. Lancet. 2010;376:875-85.

26. Swedberg K, Komajda M, Böhm M, et al. Effects on outcomes of heart rate reduction by ivabradine in patients with congestive heart failure: is there an influence of beta-blocker dose? findings from the SHIFT (Systolic Heart failure treatment with the I(f) inhibitor ivabradine Trial) study. J Am Coll Cardiol. 2012;59:1938-45.

27. Schiller NB, Shah PM, Crawford M, et al. Recommendations for quantitation of the left ventricle by two-dimensional echocardiography. American Society of Echocardiography Committee on Standards, Subcommittee on Quantitation of Two-Dimensional Echocardiograms. J Am Soc Echocardiogr. 1989;2:358-67.

28. Ekman I, Chassany O, Komajda M, et al. Heart rate reduction with ivabradine and health related quality of life in patients with chronic heart failure: results from the SHIFT study. Eur Heart J. 2011;32:2395-404.

29. Tardif JC, O'Meara E, Komajda M, et al. Effects of selective heart rate reduction with ivabradine on left ventricular remodelling and function: results from the SHIFT echocardiography substudy. Eur Heart J. 2011;32:2507-15.

30. Volterrani M, Cice G, Caminiti G, et al. Effect of Carvedilol, Ivabradine or their combination on exercise capacity in patients with Heart Failure (the CARVIVA HF trial). Int J Cardiol. 2011;151:218-24.

31. Cice G, Di Benedetto A, D'Andrea A, et al. Heart rate as independent prognostic factor for mortality in normotensive hemodialysed patients. J Nephrol. 2008;21(5):704-12.

32. Cice G, D'Isa S. Beta-blockers in CHF: kidney's consequences. G Ital Nefrol. 2006; Jan-Feb;23 Suppl 34:S51-6. 